\title{
LV-DB-1
}

\section{Upfront surgery}

\author{
Jong Man KIM ${ }^{*}$
}

Department of Surgery, Samsung Medical Center, Sungkyunkwan University School of Medicine, Seoul, Korea

\section{Introduction}

Hepatocellular carcinoma (HCC) with portal vein tumor thrombosis (PVTT) is a worst prognostic factor for HCC, resulting in a median survival time of two to 12 months, and is regarded as a contraindication for LT [1]. Sorafenib is the only recommended treatment for advanced HCC according to the Barcelona Clinic Liver Cancer (BCLC) staging system. However, the outcome of sorafenib-alone treatment is still poor, while advanced HCC is relatively common with a $10 \%$ to $40 \%$ prevalence rate in total HCC population [2,3]. As locoregional therapy develops and good outcomes are achieved with advanced HCC, an increasing variety of downstaging procedures for advanced HCC followed by surgical liver resection or liver transplantation are being performed.

\section{Surgical resection of HCC with PVTT}

Although the recommended treatment for HCC with PVTT in BCLC classification is sorafenib, some studies have shown the better overall survival (OS) for surgical operation as compared with sorafenib therapy. This aggressive treatment must be individualized to the patient's risk and tumor types [4]. Depending on the location of PVTT and operator's preference, three surgical treatments are usually adopted: hepatectomy for tumors with ipsilateral PVTT, en-bloc resection followed by portal vein reconstruction for tumors with PVTT extending beyond portal vein bifurcation, and thrombectomy for tumors with PVTT extending beyond portal vein bifurcation [5]. Surgical resection is sometimes combined with other treatments such as adjuvant TACE, neoadjuvant RT, TARE, or concurrent chemoradiation therapy (CCRT) to prevent thrombus dissemination, lower the recurrence rate, and enhance the chance of survival [69].

\section{Liver transplantation with or without downstaging for HCC with PVTT}

Liver transplantation (LT) has been also not recommended due to the high recurrence rate of HCC with PVTT, similarly to surgical resection in traditional guidelines. Furthermore, LT carries the ethical consideration of applying liver grafts to the most appropriate recipients due to the shortage of liver donors. However, LT medical technology is gradually improving for patients with HCC and many centers are attempting LDLT for advanced HCC nowadays. LDLT may lower the burden of graft shortage relative to deceased donor LT.

Previous study reported relatively good one-, three-, and five-year recurrence-free survival (RFS) rates of 63.9\%, 45.5\%, and 45.5\% and one-, three-, and five-year OS rates of $72.7 \%, 63.6 \%$, and 63.6\%, respectively [10]. With increasing outcomes of various locoregional therapies on HCC with PVTT, additional studies are more focused on LT after downstaging for HCC with PVTT. Another study covered five patients with HCC and Vp1-3 PVTT who received LT after downstaging involving 3D-CRT after TACE [11]. Here, the interval between TACE and 3D-CRT was usually two weeks (one patient did not receive TACE) and the median RFS and OS lengths were 350 and 1,055 days, respectively. This study also compared outcomes among 10 patients who received RT-alone without LT by propensity score-matching, revealing significant differences in median OS times $(1,055$ vs. 367 days; $p<0.01)$.

\section{Conclusion}

Surgical liver resection or liver transplantation after downstaging is increasingly worth considering for HCC with PVTT. Although these trials are not of excellent quality, this approach still shows better outcomes than sorafenib-alone treatment. Due to the small 


\section{AHBPS}

number of existing studies, more trials and evidence are needed before stronger conclusions can be drawn.

\section{REFERENCES}

1. Minagawa M, Makuuchi M. Treatment of hepatocellular carcinoma accompanied by portal vein tumor thrombus. World J Gastroenterol 2006;12:7561-7567.

2. Lee YH, Hsu CY, Huang YH, Hsia CY, Chiou YY, Su CW, et al. Vascular invasion in hepatocellular carcinoma: prevalence, determinants and prognostic impact. J Clin Gastroenterol 2014;48:734-741.

3. Shen J, Wen J, Li C, Wen T, Yan L, Li B, et al. The prognostic value of microvascular invasion in early-intermediate stage hepatocelluar carcinoma: a propensity score matching analysis. BMC Cancer 2018;18:278.

4. Chan SL, Chong CC, Chan AW, Poon DM, Chok KS. Management of hepatocellular carcinoma with portal vein tumor thrombosis: Review and update at 2016. World J Gastroenterol 2016;22:7289-7300.

5. Chok KS, Cheung TT, Chan SC, Poon RT, Fan ST, Lo CM. Surgical outcomes in hepatocellular carcinoma patients with portal vein tumor thrombosis. World J Surg 2014;38:490-496.

6. Peng BG, He Q, Li JP, Zhou F. Adjuvant transcatheter arterial chemoembolization improves efficacy of hepatectomy for patients with hepatocellular carcinoma and portal vein tumor thrombus. Am J Surg 2009;198:313-318.

7. Ye JZ, Wang YY, Bai T, Chen J, Xiang BD, Wu FX, et al. Surgical resection for hepatocellular carcinoma with portal vein tumor thrombus in the Asia-Pacific region beyond the Barcelona Clinic Liver Cancer treatment algorithms: a review and update. Oncotarget 2017;8:93258-93278.

8. Pracht M, Edeline J, Lenoir L, Latournerie M, Mesbah H, Audrain O, et al. Lobar hepatocellular carcinoma with ipsilateral portal vein tumor thrombosis treated with yttrium-90 glass microsphere radioembolization: preliminary results. Int J Hepatol 2013;2013:827649.

9. Lee HS, Choi GH, Choi JS, Kim KS, Han KH, Seong J, et al. Surgical resection after down-staging of locally advanced hepatocellular carcinoma by localized concurrent chemoradiotherapy. Ann Surg Oncol 2014;21:3646-3653.

10. Lee KW, Suh SW, Choi Y, Jeong J, Yi NJ, Kim H, et al. Macrovascular invasion is not an absolute contraindication for living donor liver transplantation. Liver Transpl 2017;23:19-27.

11. Choi JY, Yu JI, Park HC, David Kwon CH, Kim JM, Joh JW, et al. The possibility of radiotherapy as downstaging to living donor liver transplantation for hepatocellular carcinoma with portal vein tumor thrombus. Liver Transpl 2017;23:545-551. 